\title{
Human Amniotic Epithelium as an Unlimited Source of Oct4-Expressing Totipotent Stem Cell Subset
}

\author{
T. Echigoya ${ }^{1,2,3}$, M. Takeuchi ${ }^{1}$, H. Hori ${ }^{3}$, F. Hirahara ${ }^{2}$ and S. Yasumoto ${ }^{1, *}$ \\ ${ }^{1}$ Laboratory of Molecular Cell Biology and Oncology, Kanagawa Cancer Center Research Institute; ${ }^{2}$ Yokohama City \\ University School of Medicine and ${ }^{3}$ Hori Maternity Hospital, Japan
}

\begin{abstract}
We have explored Oct4-positive cells residing in the human amniotic membrane (HAM) at various stages of gestation. Here, we report that a number of Oct4-positive human amniotic epithelial (HAE) cells can be recovered as potentially pluripotent stem cells from any stage of human embryonic development. We found that Oct4-expressing cell subsets are clustered in specific regions of the human amniotic epithelium. Immunohistochemical examination suggests the expression levels of Oct4 protein vary at the single cell level in these cohorts as well as in vitro. These Oct4-positive HAE cells can be harvested and enriched in vitro as a potential source of pluripotent somatic stem cells under defined culture conditions. These cultured HAE cells expressed several marker genes for undifferentiated human embryonic stem (huES) cell lines that included connexins (hCx43 and hCx45), Pro-Galanin, Nanog, ABCG2 and hRex1. Several differentiation markers were also detected, such as nestin as a marker for neuroectodermal progenitor cells, albumin and amylase of hepatic cell type, and CK19 as an epithelial cell marker, as determined by RT-PCR and immunocytochemical analysis. Moreover, chromogranin A and glut2, marker genes for neuroendocrine pancreatic cells, were shown to be inducible at the mRNA level in response to glucose and nicotinamide. These results further strengthen the notion that the human amniotic epithelium provides an unlimited promising source of potentially useful hES-like somatic cells that possess multiple differentiation potential to give rise to a functionally different wide variety of cell types.
\end{abstract}

\section{INTRODUCTION}

Oct4 is a POU Domain containing octamer - binding transcriptional regulatory factor that is differentially expressed in early embryonic cells [1-3]. Pluripotent embryonic stem (ES) cell lines that possess indefinite proliferative capacity can be established from the Oct4-positive undifferentiated inner cell mass (ICM) in blastocysts [4-6]. The expression level of Oct4 protein has been demonstrated to be a critical factor for the fate determination of embryonic cells, including ES cells in vitro, which give rise to various cell types [7-9]. When its expression is downregulated, Oct4-negative cells differentiate to almost all somatic cell lineage [9-11] and the expression of Oct4 is restricted to primordial germ cells during embryonic development $[3,7,8,12-14]$. Thus, Oct4 protein has become a widely accepted specific marker molecule for cells that possess pluripotency $[5,11,14]$. Taking into account the properties exhibited by Oct4-positive embryonic cells, manipulation of human blastocysts has been eagerly claimed as a means to establish ES cell lines for use in biomedical applications $[5,15,16]$; however the manipulation of human embryos for the sake of ES cell research is still controversial and under strict regulation in many nations.

More recently, it was demonstrated that an Oct4-expressing cell subset is present within amniotic fluid [17], in which various cell types of different embryonic / fetal origin from all three germ layers, including the amniotic membrane, have been identified [18-21]. These observations raise the possibility that pluripotent somatic stem cells reside within the

*Address correspondence to this author at the Laboratory of Molecular Cell Biology and Oncology, Kanagawa Cancer Center Research Institute 2410815 Nakao, Asahi-ku, Yokohama, Japan; E-mails: kxkbk831@ybb.ne.jp, yasumoto@gancen.asahi.yokohama.jp amniotic membrane, as well as in developing embryonic tissues. In these studies, however, the recovery of Oct4positive cells from amniotic fluid was inefficient (fewer than $0.1-0.5 \%$ of recovered human amniotic fluid cells) [17], with the exact origin and biological capabilities of the various cell types found in the amniotic fluid not precisely known as yet.

Here, we have explored a resident Oct4-expressing cell subset in human embryonic tissues and demonstrate that Oct4-positive cells are present at a specific location in HAM. We have characterized these Oct4-expressing HAE cells in vitro and found that they possess self-amplifying potentials. Gene expression profiles also demonstrate the presence of several undifferentiated cell markers associated with human ES cell lines [22] as well as of multiple differentiation markers for neuroectodermal and neuroendodermal cell types. These results may provide a basis for an unlimited source of putatively pluripotent stem cells from HAM of newborns with fewer ethical barriers.

\section{RESULTS}

\section{Oct-4 Expressing Cell Subset in Human Amniotic Epithelial Cells}

Immunohistochemistry was used to identify a resident Oct4-positive cell subset within HAM obtained at full-term (40 weeks' gestation) delivery. A number of Oct4-positive cells were found to reside within a specific region of the HAE layer around the placenta, termed the stem cell niche (SCN), in which a cluster of HAE cells was found expressing Oct4 at various levels and Oct4-negative cells (Fig. 1A). The varied expression levels of Oct4-protein were analyzed by software to measure pixel intensities of the DAB stain per 
cell indicative of strong $(s)$, moderate $(m)$, weak $(w)$ and negative $(n)$ within the overall collection of Oct4-positive cells (Fig. 1A a - c). These Oct4-positive cell subsets were distributed across the HAE layer, but were rarely found in far-distal regions of the SCN (data not shown).

This raises the intriguing possibility that the pluripotent Oct4-expressing stem cell subset may be isolated from the human amnion with a high degree of efficiency, with consequently fewer problems as compared to the generation of equivalent cells from the ICM of developing human embryos.

\section{Oct4-Positive HAE Cells can be Propagated In Vitro}

To investigate the characteristics of Oct4-positive HAE cells, we firstly attempted to recover HAE cells from amniotic tissue specimens obtained after full - term derivery (av.40 wgestation) and to grow them in vitro. We utilized a mixtur of two media types (DMEM and Ham's MCDB153 with supplements, details in Methods section) to facilitate the growth in vitro; this permitted expansion of HAE cells over a $6 \mathrm{w}$ period (Fig. 1B). Our culture media mixture effectively supported the growth of HAE cells in contrast to conven-
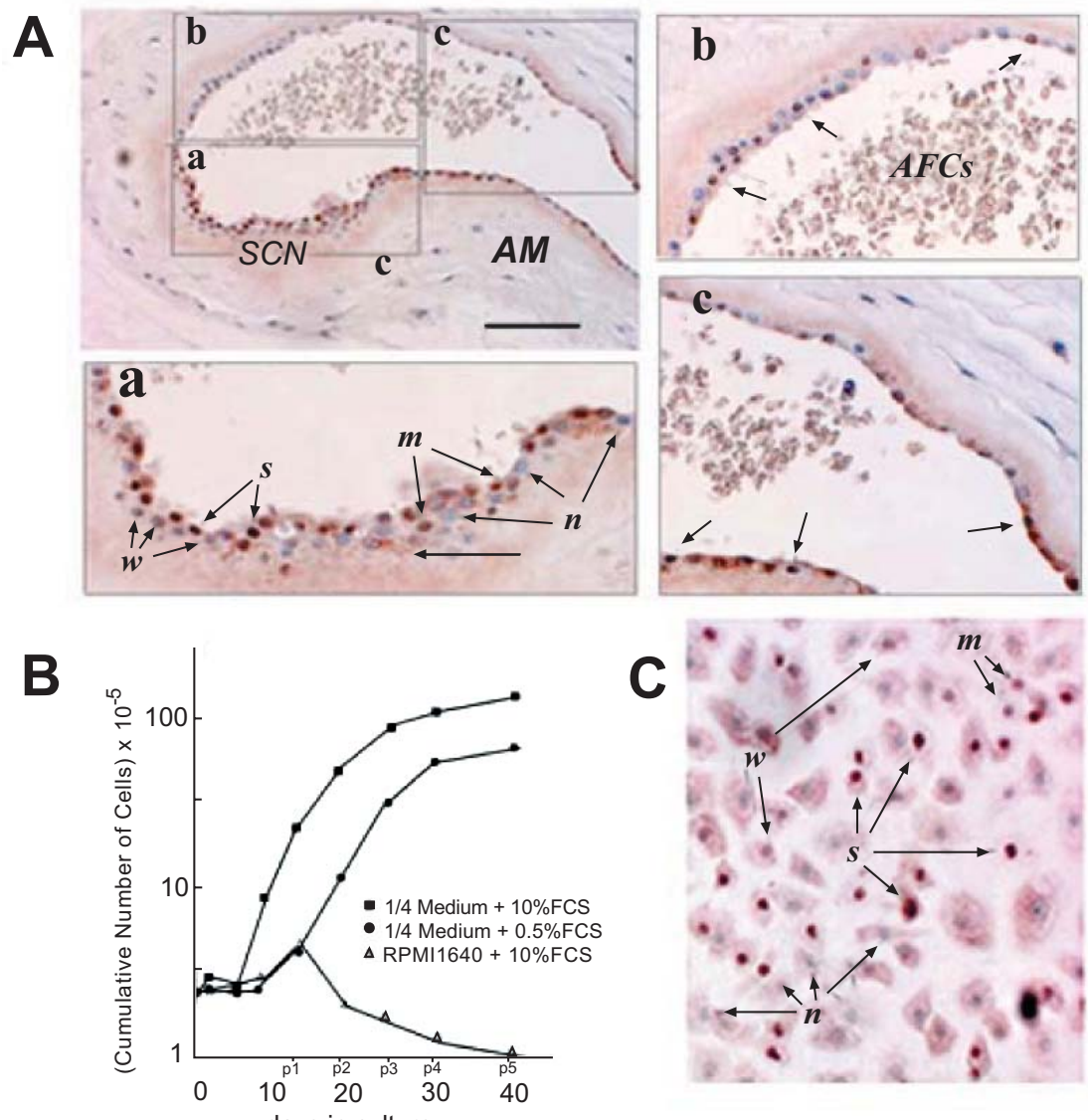

B
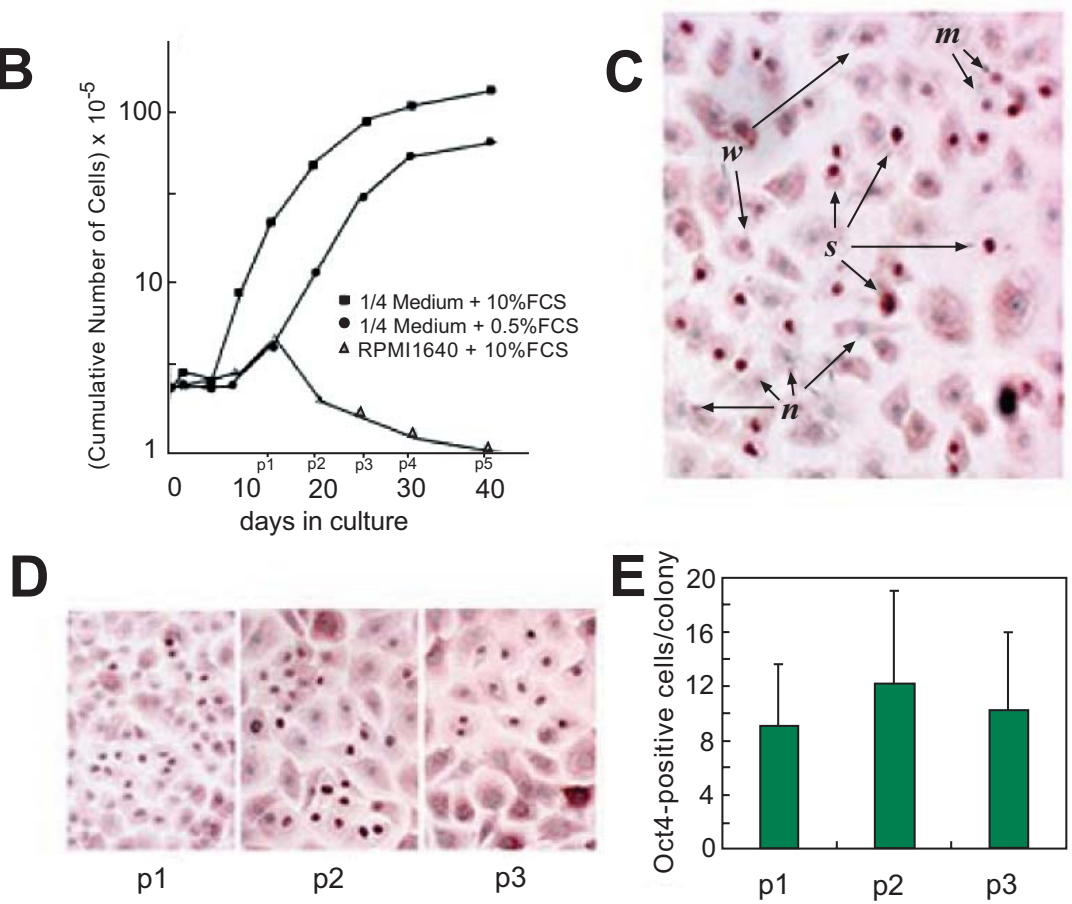

Fig. (1). Oct4-positive cell subsets detected in the HAE layer and within its cultured HAE cell population derived from full-term (40 weeks) delivery. All specimens were briefly counter-stained with hematoxylin unless otherwise indicated. A) Immunohistochemically detected Oct4positive cell subsets (brownish colour) are clustered at a local HAE layer around the placenta, termed SCN. Bar indicates $100 \mu \mathrm{m}$. Boxed regions $(\mathrm{a}, \mathrm{b}, \mathrm{c})$ correspond to the magnified panels, $\mathrm{a}, \mathrm{b}$ and $\mathrm{c}$, respectively. AM: amniotic mesoderm. Panel a, a number of Oct4-positive cells cluster within the mass of HAE cells. Arrows $s$ : strongly positive, $m$ : moderately positive, $w$ : weakly positive, $n$ : negative. Bar indicates $100 \mu \mathrm{m}$. Arrows in panels $\mathbf{b}$ and $\mathbf{c}$ indicate Oct4-positive subsets residing at the basal layer that express Oct4 protein at relatively higher expression levels. AFCs: amniotic fluid cells. B) Growth of HAE cells during serial cultivation in different culture media. p1 - p5 indicate the day of passage. $C$ ) Immunocytochemical detection of Oct4-positive HAE cells in the primary culture of 40-week (w) HAE cells. $s, m$ and $w$ indicate levels of Oct4 positvity as strong, moderate and weak, respectively. $n$ indicates Oct4-negative. $\boldsymbol{D}$ ) The growing Oct4-positive cells that form microcolonies among the Oct4-negative HAE cell population. p1: primary culture, p2: secondary culture, p3: tertiary culture. (see details in the text). $\boldsymbol{E})$ Average number of Oct4-positive cells within the collected Oct4-positive colonies $(\mathrm{n}=12)$. 
tional RPMI1640-based media containing 10\% FCS; however the Hae cells subsequently ceased growing after several passages by splitting at 1:3 and could not be expanded thereafter.

We therefore next examined whether Oct4-positive cells could be recovered and maintained in vitro. Immunohistochemical examination of cultured Hae cells demonstrated that Oct4-positive cells present within the overall population of HAE cells obtained from $40 \mathrm{w}$ gestation specimen (Fig.1C). Moreover, we noticed that the expression level of Oct4-protein varied within individual Oct4-posirive cells, as noted previously among HAE layer. They were similarly recognized as Oct4-positive cells that were expressed stronger $(s)$, moderately $(m)$ and weakly $(w)$ (Fig.1C, arrows).

To follow the fate of Oct4-positive cells in serial cultivation in vitro, the cultured HAE cells were immunostained with anti-Oct 4 at the end of each passage when the cells became confuent. We found that Oct4-positive cells persistently remained in the serial cultivation in vitro. Moreover, Oct4-positive HAE cells appeared to clonally grow and form micro-colonies consisting of Oct4-positive cells among the Oct4-negative cells, strongly suggesting that Oct4-positive HAE cells are capable of self-amplifying to some extent under these culture conditions (Fig. 1D). When 12 microcolonies, consisting of more than seven Oct4-positive cells, were randomly selected and the number of Oct4-positive cells at the time of each passage was averaged (primary to tertiary) (Fig. 1E). These results may allow us to roughly estimate the division potential as about once a few days, indicating that Oct4-expressing HAE cells maintained their division potentials throughout serial cultivation, at least up to a month.

\section{Oct4-Expressing HAE Cells in the Earlier Stages of Hu- man Fetus Development}

We next examined whether Oct4-expressing cells could similarly reside within the amniotic membrane in earlier fetus development. Immunohistochemistry of HAM at 16 - 18 w gestation, clearly showed that Oct4-positive cells existed within the HAE layer where Oct4-positive cells were rather uniformly distributed over the HAE layer (Fig. 2A). The HAE cells recovered from these specimens actively grew under our culture conditions. Immunocytochemistry again identified an Oct4-positive HAE cell cohort within the Oct4negative HAE cell population (Fig. 2B). Moreover, the finding of mitotic figures of Oct4-positive cells directly shows that Oct4-expressing HAE cells possess division potentials in vitro (Fig. C, arrow). Notably, post-mitotic nuclei were both equally Oct4-positive, strongly suggesting that Oct4expressing HAE cells possess self-renewing activity with respect to the Oct4 expression that results in the amplification of Oct4-positive stem cells.

\section{Proliferation Potentials and Stemness-Related Gene Ex- pression}

When the cell growth of 18 - w HAE cells was compared with that of $40-$ w HAE cells, the HAE cells obtained from the earlier embryonic stage exhibited superior multiplication potential (Fig. 2D) and contained more Oct4-positive cells in the culture (Fig. 2E).

However, the number of Oct4-positive cells decreased to some extents during serial cultivation (data not shown), with the proportion of Oct4-negative cells accumulating within the growing HAE cell population over time, which may be attributable to the onset of differentiation in vitro.

To know whether gene expression profiles would change in HAE cells derived from different ages, $18 \mathrm{w}$ versus $40 \mathrm{w}$, of amnion, a unique set of marker genes that is associated with undifferentiated human ES cells was examined by RTPCR using the published primer sets (see MATERIALS and METHODS). We detected mRNAs of several genes that have been identified within certain human ES cell lines [22]. These included connexins ( $\mathrm{hCx} 43, \mathrm{hCx} 45)$, Pro-Galanin, Nanog, ABCG2 and hRex1 (Fig. 2F). While, hUTF, Dppa5, GDF3 and hTERT were undetecteble at mRNA level (data not shown). These results demonstrate that HAE cells contain undifferentiated and potentially pluripotent stem cells throughout human fetus development.

\section{Marker Genes Relevant to Multiple Differentiation Po- tentials of HAE Cells}

To characterize differentiated HAE cells in vitro, the expression of several cell-specific differentiation markers were examined at the mRNA level, with transcript for nestin, albumin, CK19 and Oct4 being assessed by RT-PCR analysis (Fig. 3A). These results suggest that the cultured HAE cells became constituted of heterogeneous cell subsets, as a result of differentiation to nerve cells or hepatocytes in vitro. Nestin mRNA was only present at a low abundance; moreover, the level of nestin mRNA remain unchanged in HAE cells at different developmental stages, i.e. $16 \mathrm{w}$ versus $40 \mathrm{w}$ gestation. It is consistent with the observation that only a few percent of nestin-positive cells were shown by immunocytochemistry (Fig. 3B a). In contrast, the expression levels of Oct 4 mRNA in HAE cells from 16 w HAE layer were several fold higher than that found in $40 \mathrm{w}$ samples, probably reflecting differences in the number of Oct4-expressing cells within the HAE cell population (refer to Fig. 2). Immunofluorescent staining also identified an albumin-producing cell subset (Fig. 3B, b), suggesting the presence of hepatic cells. Confocal laser immunofluorescent microscopy further demonstrated that the albumin-positive cells also co-expressed cytokeratin 19 (CK19), which is a marker protein for epithelial cells including bile ductal epithelial cells (Fig. 3B. b, c, d).

\section{Specific Cell Markers for Neuroendocline Cells}

To test for other differentiation markers, we attempted to identify pancreatic cell-specific gene expression. However, neither insulin nor glucagon was detected, thus providing no evidence for terminally differentiated pancreatic $\beta$ cells and $\alpha$ cells under the present culture system (data not shown). Instead, we detected the expression of $\mathrm{CgA}$ and glut2, which are also markers for neuroendocrin cells (Fig. 3C). These latter two genes were detected after being induced with higher concentrations of glucose in the presence of nicotinamide. Moreover, the augmented expression of both $\mathrm{CgA}$ and glut2 mRNA transcripts was detected after incubating for several days in culture, suggesting that a commitment toward pancreatic differentiation proceeded with time in culture (Fig. 3C). However, these responses to glucose were not always consistent in the expressions of $\mathrm{CgA}$ and glut 2 in different HAE cell specimens. Augmented expression levels 

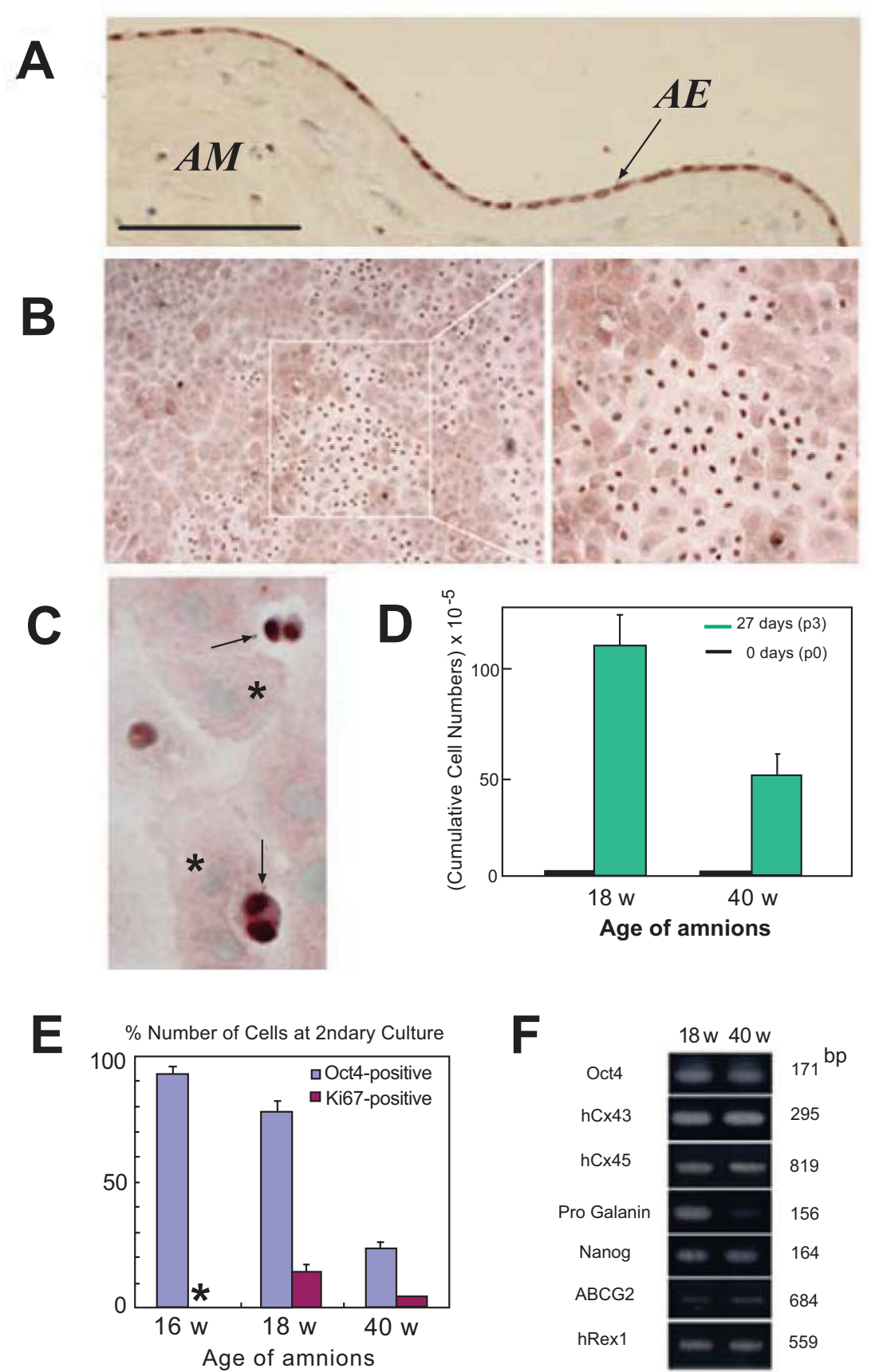

Fig. (2). Comparative growth potentials of Oct4-positive HAE cells derived from different gestation periods. A) Immunohistochemically detected Oct4-positive HAE cells over the HAE layer of 18 - w gestation. Bar indicates $100 \mu \mathrm{m}$. AM, Amniotic mesoderm. AE, Amniotic epithelium $\boldsymbol{B}$ ) Oct4-positive cells within 18 - w HAE cells in primary culture for 2 weeks. Right panel shows the magnified area indicated by a white box in the left panel where Oct4-positive cells are clustering. $\boldsymbol{C}$ ) Symmetric cleavage pattern of Oct4-positive cells in vitro. Arrows indicate post - mitotic figures of Oct4-positive cells in the primary culture of 18 - w HAE cells. * indicates Oct4-negative HAE cells within the interphase in the cell cycle $\boldsymbol{D}$ ) Comparison of the in vitro multiplication potential of HAE cells obtained from amnions of 18 - w and 40 $\mathrm{w}$ gestation. Cells were plated in a collagen IV-coated culture dish at a concentration of $1 \times 10^{5} / \mathrm{cm}^{2}$, followed by serial cultivation. Cumulative cell numbers over a month were compared. $\boldsymbol{E}$ ) Recoveries of Oct4-positive cells in the cultured HAE cells derived from various gestation periods. Cycling HAE cells were determined by the immunostaining using anti-human Ki-67 monoclonal antibody (clone B56, BD Biosciences). * Data not available. $\boldsymbol{F}$ ) The expression of various marker genes related to undifferentiated human ES cells in the HAE cells as detected by 35 cycles of RT-PCR (see details for the primer sets in the text).

of CgA was much higher than that of glut2 in HAEa cells. In contrast, glut 2 was much more sensitive to be induced by higher doses of glucose in HAEb cells. Differential levels of the induced mRNA of $\mathrm{CgA}$ and glut 2 by high concentrations of glucose $(35 \mathrm{mM})$ were detected in these two different specimens in culture for a week. - and + indicate concentrations of added glucose as $5 \mathrm{mM}$ and $35 \mathrm{mM}$, respectively, in the presence of nicotinamide $(10 \mathrm{mM})$. We also detected amylase gene expression. There are known to be three isozymes specific for salivary, pancreatic and hepatic cell types in 

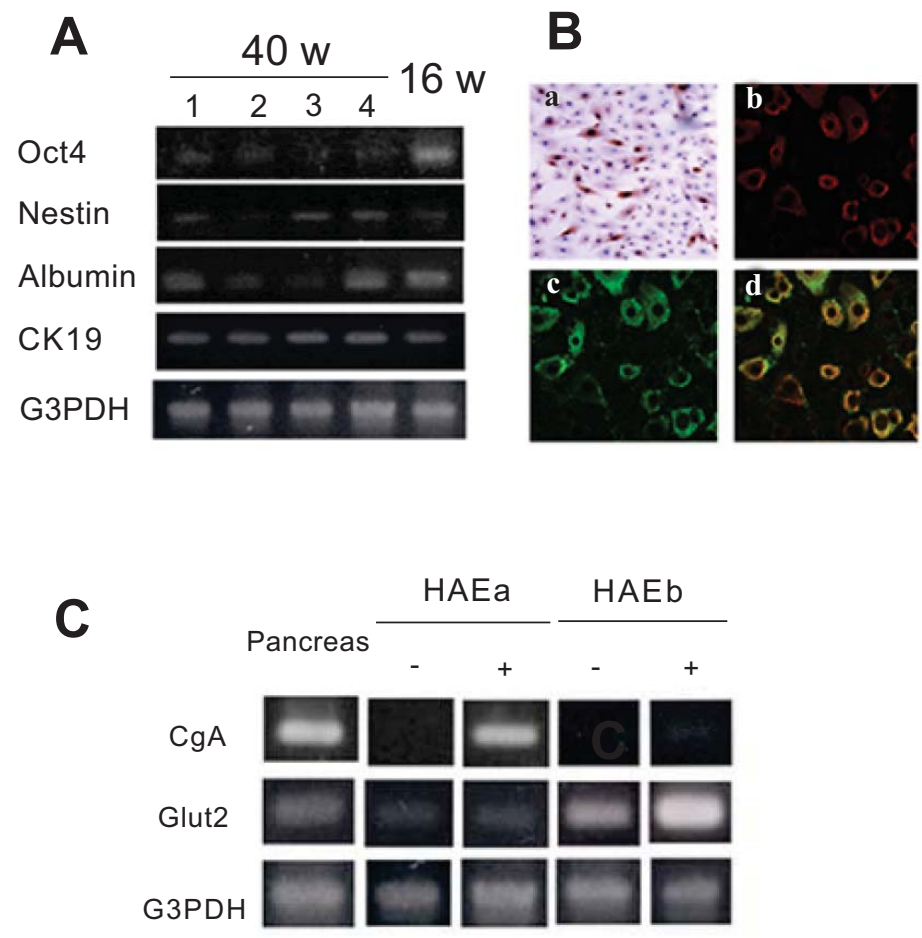

Fig. (3). Detection of various cell-type specific marker molecules expressed in the cultured HAE cells. $\boldsymbol{A}$ ) RT-PCR analysis of mRNA species expressed in the HAE cells obtained from 4 individual specimens at $40 \mathrm{w}$-gestation (lanes 1-4) and from one $16 \mathrm{w}$ specimen. B) (a) Immunocytochemical detection of nestin. $b$ and $c$, Detection of albumin producing cells by immunofluorescence confocal laser microscopy. $b$. CK19 (Cy3-Red, excitement $543 \mathrm{~nm}$; emission >560 nm). c, albumin (Alexa-Green, excitement $488 \mathrm{~nm}$; emission > $505 \mathrm{~nm}$ ). d, Colocalization b and c (merged-Yellow). $\boldsymbol{C}$ ) The induced expression of pancreatic cell markers, $\mathrm{CgA}$ and glut2, in cultured HAE cells (HAEa and HAEb) in response to glucose as detected by RT-PCR. Pancreas: total RNA of human pancreas (Biochain Institute, Inc). HAEa and $\mathrm{HAEb}$ were derived from different specimens of the $40 \mathrm{w}$ amniotic epithelia.

humans; the corresponding genes are designated Amy1, Amy2A and Amy2B, respectively [26, 27]. Restriction digestion of PCR products by Hae II and BamH1 discriminated the hepatic type (Amy2B) from the pancreatic type (Amy2A) (Fig. 4A \& B).

Restriction digestion with three restriction enzymes identified distinctive mRNAs for isozymes of the cell-type specific amylases (pancreas, liver, salivary) [26]. The amplified cDNA(\#1 and \#3) using primers a and $c$ were cleaved by Pst1 and BamH1, but cDNA \#2 by using primer $b$ was insensitive to the HaeII digestion. These cleavage patterns of three different cDNA(\#1, \#2 and \#3) of HAE cells by the corresponding restriction enzymes indicate that the same type of amylase isoform ought to be expressing in hepatoma cell line HepG2 but neither pancreas nor salivary. Moreover, since pancreatic cDNAs amplified by all three primers consisted of heterogeneous cDNAs that represent different sensitivities to all three restriction enzymes, implicating that pancreatic amylase may contain different isoforms.

Overall, these results demonstrate that Oct4-positive HAE cells possess differentiation potentials toward various cell types belonging to neuroectodermal and neuroendocrin cells that include nerve cells, pancreatic cells, hepatic cells and biliary ductal epithelial cells.

\section{Origin and the Fate of Oct4-Positive Cell in Amniotic Membrane}

Although the fate of Oct4-positive cells within the human amnion is not exactly known, some may detach from the
HAE layer and become amniotic fluid cells as found in the amniotic cavity (Fig. 5A. arrow a; arrows b and c indicate Oct4-positive and negative HAE cell, respectively). A few of them may be remained as amniotic fluid cells and can be detectable to be Oct4-positive cells recovered from amniotic fluid (Fig. 5B, right-hand panel, arrow b; arrow a indicates Oct4-positive HAE cell). In addition to these Oct4-expressing cells within the HAE layer, Oct4-positive cells in human amniotic mesoderm were also found, albeit on a discrete level, (Fig. 5C, marking squares $a$ and $b$ ). The magnified figures for the typical area, $\boldsymbol{a}$ and $\boldsymbol{b}$, clearly showed a solitude Oct4-positive cells in the matrix of amniotic mesoderm (Fig. 5C, right-hand panels $a$ and $b$, arrows).

These immunohistochemical observations have directly demonstrated the potentially useful specific cell subsets that express Oct4 at various levels in the HAM throughout gestation.

\section{DISCUSSION}

We have demonstrated that HAM contains putatively pluripotent somatic stem cells expressing Oct4-protein. Oct4-positive cells were identified as cell subsets that differentially expressed Oct4 protein in vivo and in vitro. Oct4 is a well-known transcription factor that is expressed during the cell cleavage stage, which occurs very early during embryonic development; moreover, its expression becomes restricted to the ICM of the blastocyst that develops into pluripotent progenitors of extra-embryonic tissues [4-8, 11, 14]. Oct4 expression is then down-regulated within very early 
human embryos, and no longer detectable in almost all somatic cells by approximately 8th-9th week of gestation [10, 28]. On the other hand, its expression is maintained at high level in migrating primordial germ cells $[3,7,8,12-14]$. Regulation of Oct4 expression is associated with epigenetic modification via altered DNA methylation of the transcriptional promoter of Oct $4[29,30]$. Thereby, Oct4 is believed to be negative in almost all somatic cell lineages with hypermethylation at the transcriptional promoter, except for germ line cells and certain cell lines [31,32].

A
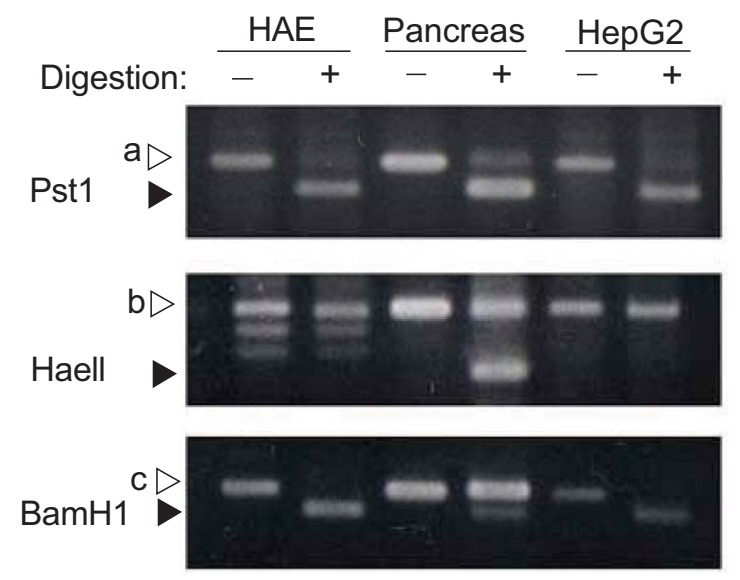

B

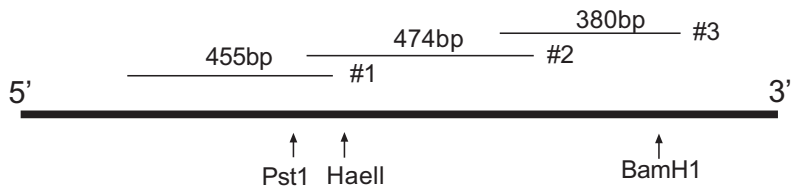

\begin{tabular}{|c|c|}
\hline Pancreas & 00 \\
\hline Liver & $0 x$ \\
\hline Salivary & $x \quad 0$ \\
\hline
\end{tabular}

Fig. (4). Hepatic-type differentiation identified by the expression of amylase isozyme mRNAs. A) Cell-type specific transcripts were determined by the restriction digestion of the cDNAs (\#1, \#2 and \#3) amplified with the specific primer sets, respectively, using Pst1, HaeII and BamH1. Each amplified cDNA from mRNAs of HAE cells, pancreas and HepG2 exhibits differential sensitivities to Pst1, HaeII and Bam H1. +, indicates enzyme treatment. B) Schematic illustration for differential cleavage patterns of the amplified $\operatorname{cDNA}(\# 1, \# 2$ and \#3) using the different set of primers (a, b and c).

In this study, we have identified the Oct4-positive cell subset that resides at a specific location within the HAE layer obtained at full-term gestation. This is the first clear evidence for a resident Oct4-expressing cell mass in extraembryonic tissue from late gestation. While it has not been clarified why HAE cells contain various cell types with a wide spectrum of differentiation, we noted in this study that the expression levels of the Oct4-protein were varied within individual Oct4-positive HAE cells. Since differential levels of Oct4 expression should be critically important for the determination of cell-fate of the pluripotent ES cells [7-9], it is likely that various differentiating cell types in the amniotic fluid were possibly derived from Oct4-expressing HAE cells $[19,20]$. Our present study may explain, at least in part, the origin of the few Oct4-positive cells that have been found within human amniotic fluid [17]. These observations might allow us to speculate as to what physiological molecules are produced by the wide variety of functional HAE cells present and which play a constructive role in the growth of various tissues and organs during fetal development. Consistently, a number of different cytokines and growth factors have been detected in culture media of HAE cells, as well as in amniotic fluid [33]. As a consequence of these characteristics, HAE cells (or HAM) could be reasonable source of implantation for clinical purposes [25, 34-36]. As no suitable

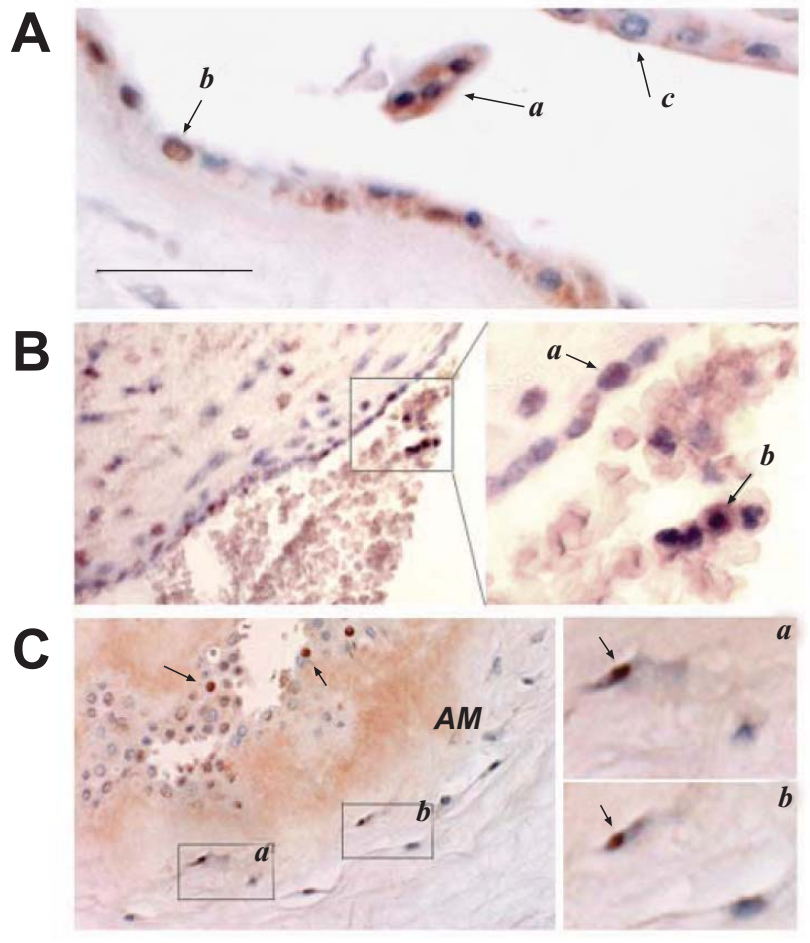

Fig. (5). Oct4-positive cells detected in not only amniotic epithelium but also amniotic mesoderm. Since these amniotic tissue specimens were briefly counter-stained with hematoxylin, the Oct4negative cell nuclei should be purple-blue, while the Oct4-positive nuclei should be brownish. A) Oct4-positive amniotic fluid cells appeared to be detached from amniotic epithelium (a). Arrows $b$ and $c$ indicate Oct4-positive and Oct4-negative HAE cells, respectively. Bar indicates $50 \mu \mathrm{m}$. B) This panel shows an Oct4-positive cell cohort within amniotic fluid cells (AFCs). Arrow $a$ indicates Oct4-positive cell resided in HAE layer. Arrow $b$ shows Oct4positive amniotic fluid cells. Note Oct4-protein localized to the nucleus. C) Oct4-positive cells detected in both HAE layer (arrows on left-hand panel) and connective tissue of HAM (arrows in the panels $a$ and $b$ ).

cell surface marker associated with Oct4-positive cells is available at this moment, we are hampered with respect to isolation of viable Oct4 expressing cells from the overall population of HAE cells.

The cultured HAE cells obtained from the earlier stage of gestation clearly show evidence that Oct4-positive HAE 
cells possess a mitotic, but not unlimited, potential in vitro. Since symmetrical cell cleavage occurs in Oct4-positive cells, Oct4-expressing HAE cells appeared to possess selfamplifying potential, implicating that the number of Oct4positive cells may maintain at constant level during full-term gestation. This speculation could be supported by the observation that Oct4-positive HAE cells were, more or less, capable of maintaining a self-amplifying potential, resulting in the formation of microcolonies consisting of a cohort of Oct4-positive cells. Selective and continued proliferation of these Oct4-expressing HAE cells may supply a unique cell system that could have pluripotent human cell lines alike ES cells, since the expression of a set of genes related to undifferentiated human ES cell lines [22] was detected in HAE cells. However, expression of catalytic subunit of telomerase (hTERT) was not detected by RT-PCR within the cultured HAE cells from any stages of gestation as yet. Since telomerase activity detectable in normal human epithelial cells is dynamically regulated and restricted to a specific cell subset that is undergoing DNA synthesis [24, 37, 38], not all Oct4positive HAE cells may have detectable telomerase activity. In fact, only a small portion of Oct4-positive cells is cycling in consistent with the presence of Ki67-positive cycling cells in vitro (refer to Fig. 2, E), but it may not be sufficient for detecting telomerase activity unless the proliferating Oct4positive cells are concentrated [24, 37, 38].

As for the differentiation potentials, we have shown a possibility for hepatic differentiation, as detected by albumin expression at the mRNA and protein levels. Moreover, since the laser confocal microscopic analysis demonstrated that albumin-producing cells were also CK19-positive, our results lead us to conclude that HAE cells may contain hepatic progenitor cells in a biliary epithelial cell lineage [39]. This process appeared to be time-dependent in culture where an albumin+/CK19+ cell subset became detectable after incubation for longer than a week in culture (our unpublished results), suggesting that HAE cells contained stem cells or progenitor cells for the hepatic cell lineage. This is further supported by the detection of hepatic-type amylase gene expression.

We have also detected other neuroendocrine cell markers, such as $\mathrm{CgA}$ and glut2, indicating that HAE cells also possess the capacity for differentiation to pancreatic cells [40]. This process also proceeded in a time-dependent manner in culture. These results strongly suggest that a multiple differentiation capacity of HAE cells could be attributable to the presence of the Oct4-expressing cell subset residing in the amnion. However, these cells failed to produce $\alpha$-FP, Insulin and glucagon, possibly due to lack of specific factors in vitro that are necessary for progression towards terminal differentiation. Although the developmental origin of Oct4positive HAE cells is not precisely known as yet, these may have been derived from ICM (epiblast) but not trophoectodermal origin, as Oct4-positive HAE cells obtained in this study represent phenotypes of neuroendodermal and neuroectodermal cell types. Thus, the fate of Oct4-positive HAE cells could be distinct from that of trophoectodermal stem (TS) cells [10, 41]. Whether Oct4-expressing cell subset could be functionally and genetically equivalent to that of $\mathrm{hES}$ cells remains to be investigated.

\section{MATERIALS AND METHODS}

\section{Preparation of Human Amniotic Epithelial Cells}

Human amniotic specimens used in this study were obtained from approximately 50 cases of full-term, averaged at 40 weeks $(40 \mathrm{w})$, and 5 cases of artificial abortion at 16-18 weeks (16-18 w) gestation that had given informed consent in Hori Maternal Hospital, Yokohama. An ethical committee of Kanagawa Cancer Center inspected and approved the preclinical investigation on amniotic cells prepared from human amniotic apparatus (Sep. 1. 2000). The amnions were kept at $4^{\circ} \mathrm{C}$ in cold HEPES-buffered saline (HBSS) until processing. The amniotic membrane was then mechanically peeled from the associated placental tissues by using a forceps, cut into smaller pieces (approximately $10 \times 10 \mathrm{~cm}^{2}$ in size) and washed several times in HBSS.

The AM fragments were incubated with Dispase solution (Sigma Co., USA) in water bath at room temperature for 3040 min followed by gentl sonication and swirling under the DM-1 sonic system (DM-1, TOSHO DENNKI, Yokohama, Japan). Clumps of HAE cells were harvested by either precipitation under $1 \mathrm{G}$ or a brief centrifugation at 500-800 rpm for a few minutes. The dissociated HAE cells as cell clumps were briefly treated with $0.025 \%$ trypsin in $0.01 \%$ EDTA solution, if necessary, for the single cell suspension. The mono-dispersed single cell suspension of HAE cells was prepared by filtration through a $100 \mathrm{~mm}$ pore size membrane, followed by centrifugation at 1,500 rpm for 5 minutes. This improvement of the cell dissociation method resulted in more efficient and specific recovery of the HAE cells and minimized possible contamination of fibrobrasts, endothelial cells and blood cells.

\section{Cell Culture}

The HAE cells were cultured in the following medium as convenient for most human epithelial cells [23, 24]; a 1:4 (v/v) mixture of Dulbecco's modified Eagle's medium (DMEM) and Ham's MCDB153 medium, respectively, supplemented with hydrocortisone $(0.2 \mathrm{mM})$, epidermal growth factor (EGF) $(10 \mathrm{ng} / \mathrm{ml})$, ethanolamine $(5 \mathrm{mM})$, O-phosphoethanolamine $(5 \mathrm{mM})$, transferrin $(10 \mathrm{mg} / \mathrm{ml})$, insulin $(5$ $\mathrm{mg} / \mathrm{ml})$, bFGF (10 $\mathrm{ng} / \mathrm{ml})$ and bovine pituitary extract $(150$ $\mathrm{mg} / \mathrm{ml})$. HAE cells were plated in collagen IV coated dish.

\section{Immunostaining and Fluorescence Microscopy}

The amnion tissue specimens were fixed with $10 \%$ formalin overnight and paraffin-embedded samples were prepared by conventional histological methods. Tissue sections of $4 \mathrm{~mm}$ thickness were processed for immunohistological examination via the streptoavidin-biotin (SAB) method. Oct4 was detected by mouse monoclonal antibody (clone C10, SC-5279; Santa Cruz Biotechnology; http://www.scbt. $\mathrm{com} /$ ). Tissue sections were counterstained with hematoxylin. All antibodies used in this study were diluted by phosphate buffered saline (PBS) to an appropriate ratio as indicated in parentheses following each antibody, unless otherwise specified.

As for culture samples for staining nestin and Oct4, the cells were fixed with $10 \%$ formalin for 30 minutes and permeabilized with $0.2 \%$ Triton $\mathrm{X}-100$ for 5 minutes, followed by incubation in $0.3 \% \mathrm{H}_{2} \mathrm{O}_{2}$ for 30 minutes. The cells were 
Table 1. The List of PCR-Primers Used in this Study

\begin{tabular}{|c|}
\hline nestin : (forward, AGAGGGGAATTCCTGGAG; reverse, CTGAGGACCAGGACTCTCTA) \\
\hline Oct 4 : (forward, GAAGCTGGAGAAGGAGAAGCTG; reverse, CAAGGGCCGCAGCTTACACACATGTTC) \\
\hline cytokeratin(CK)19 : (forward, ATGAGGAGGAAATCAGTACG; reverse, TCCAAGGCAGCTTTCATGCT) \\
\hline albumin : (forward, CGTCGAGAGCACACAAGAG; reverse, CAGCAGTCAGCCATTTCACC) \\
\hline glut-2 : (forward, CCTGTTTATGCAACCATTGG; reverse, GCAGCAGGACGTGGTCCTTG) \\
\hline ChromograninA : (forward, CCGCTGTCCTGGCTCTTCT; reverse, CCGCTGTGTTTCTTCTGCTG), [25] \\
\hline hCx43 : (forward, TACCATGCGACCAGTGGTGCGCT; reverse, GAATTCTGGTTATCACGGGGAA), [22] \\
\hline hCx45 : (forward, CTATGCAATGCGCTGGAAACAACA; reverse, CCCTGATTTGCTACTGGCAGT), [22] \\
\hline Pro-galanin : (forward, AAGGAAAAACGAGGCTGGAC; reverse, GGACCTGTCAAAGCTTCCTG), [22] \\
\hline Nanog : (forward, AGTCCCAAAGGCAAACAACCCACTTC; reverse, ATCTGCTGGAGGCTGAGG TATTTCTGT CTC), [22] \\
\hline ABCG2 : (forward, GTTTATCCGTGGTGTGTCTGG; reverse, CTGAGCTATAGAGGCCTGGG), [22] \\
\hline hRex 1 : (forward, TGAAAGCCCACATCCTAACG; reverse, CAAGCTATCCTCCTGCTTTGG), [22] \\
\hline amylase \#1 : (forward, GCTGTGAGTGCAGGAACAAG; reverse, TTGAATTCAGTCACCCGGCC), [26] \\
\hline amylase \#2 : (forward, AATTGATCTGGGTGGTGAGC; reverse, CTTATTTGGCGCCATCGATG), [26] \\
\hline amylase \#3 : (forward, ACTTGTGGCAATGACTGG; reverse, GAGGATATGCATTTAATTTT), [26] \\
\hline
\end{tabular}

incubated with a rabbit anti-nestin polyclonal antibody (H85, SC-20978, Santa Cruz Biotechnology) and a goat antiOct-3/4 polyclonal antibody (1:200) (C-20, SC-8629, Santa Cruz Biotechnology). Immunocytochemistry was performed by the SAB method using the Histofine SAB-PO kit (Nichirei;http://www.nichirei.co.jp/bio/english/index.html). Visualization of antibody-antigen complex was accompl ished using a nickel-intensified 3, 3'-diaminobenzidine reaction (Sigma-Aldrich; http://www.sigmaaldrich.com/Local/ SA_Splash.html).

To carry out double-staining for CK19 and albumin, cultured cells were incubated in PBS for 1 hour, fixed with $4 \%$ paraformaldehyde for 10 minutes and permeabilized with $0.2 \%$ Triton X-100 for 5 minutes. The slides of fixed cells were then washed three times with PBS-0.2\% Tween and blocked with Dako Protein Block Serum Free (DakoCytomation; http://www.dakocytomation-japan.jp/) for 5 minutes. The following primary antibodies, with respective dilutions in parentheses, were used: mouse anti-human CK19 monoclonal (1:100) (Sigma-Aldrich) and sheep anti-human albumin polyclonal (1:50) (Serotic; http://www.serotec.com/asp/ index.html). The respective secondary antibodies used were Cy3-conjugated goat anti-mouse IgG (1:100) (Jackson Immuno Research Laboratory.; http://www.jacksonimmuno. com/) and Alexa Fluor 488 donkey anti-sheep IgG (1:200) (Molecular Probes; http://www.probes.com/). Immunofluorecent images were obtained under confocal laser microscope (LSM 5 PASCAL, Axiovert 200M, Zeiss; http://www. zeiss.com/).

\section{RT-PCR and Primers}

Total RNA was extracted from cultured HAE cells using TRIZOL (Invitrogen; http://www.invitrogen.com/content. cfm?pageid=13) following the manufacturer's instructions. Target genes were amplified by PCR using the following primer pair combinations (Table $\mathbf{1}$, reference number for primers previously reported were indicated by the number of references). Amplified RT-PCR products were fractionated on Tris-Borate-EDTA $2 \%$ agarose gels and visualized under ultraviolet light in the presence of ethidium bromide.

\section{ACKNOWLEDGEMENTS}

This work was supported in part by Grants-in Aids for the Ministry of Science Technology and Sport, and Cell Bank from the Ministry of Health, Labor and Welfare to S.Y. Authors thank Drs, Morimura S, Kikuchi K and Saiga, $\mathrm{K}$ for a number of technical supports and advices throughout this study.

\section{REFERENCES}

[1] Scholer HR, Balling R, Hazopoulos AK, et al. Octamer binding proteins confer transcriptional activity in early mouse embryogenesis. EMBO J 1989; 8: 2551-2557.

[2] Okamoto K, Okazawa H, Okuda A, et al. A novel octamer binding tranascription factor is differentially expressed in mouse embryonic cells. Cell 1990; 60: 461-472.

[3] Rosner MH, Vigano MA, Ozato K, et al. Pou-domain transcription factor in early stem cells and germ cells of the mammalian embryo. Nature 1990; 345: 686-692.

[4] Nicols J, Zevnik K, Niwa H. Formation of pluripotent stem cells in the mammalian embryo depends on the POU transcription factor Oct4. Cell 1998; 95: 379-391.

[5] Reubinoff BE, Pera MF, Fong CY, et al. Embryonic stem cell lines from human blastocysts: somatic differentiation in vitro. Nat Biotechnol 2000; 18: 399-404.

[6] Rossant J. Stem cells from the mammalian blastocyst. Stem Cell 2001; 19: 477-482.

[7] Palmieri S, Peter W, Hess H. Oct4 transcription factor is differentially expressed in the mouse embryo during establishment of the first two extraembryonic cell lineages involved in implantation. Dev Biol 1994; 166: 259-267.

[8] Pesce M, Wang X, Wolgemuth D, et al. Differential expression of the Oct4 transcription factor during mouse germ cell differentiation. Mech Dev 1998; 71: 89-98. 
[9] Niwa H, Miyazaki J, Smith AG. Quantitative expression of Oct-3/4 defines differetiation, dediffretiation or self-renewal of ES cells. Nat Genet 2000; 24: 372-376.

[10] Hay DC, Sutherland L, Clark J, et al. Oct-4 Knockdown Induces Similar Patterns of Endoderm and trophoblast diffrentiation markers in human and mouse embryonic stem cells. Stem Cell 2004; 22: 225-235.

[11] Pesce M, Scholer HR. Oct-4: gatekeeper in the beginnings of mammalian development. Stem Cells 2001; 19: 271-278.

[12] Scholler HR, Hatzopoulos AK, Balling R, et al. A family of octamer specific proteins present during mouse embryogenesis: evidence for germ line-specific expression of an Oct factor. EMBO J 1989; 8: 2543-2550.

[13] Scholer HR, Dressler GR, Balling R, et al. Oct-4: a germlinespecific transcriptionfactor mapping to the mouse t-complex. EMBO J 1990; 9: 2185-2195.

[14] Donovan PJ, Gearhart J. The end of the beginning for pluripotent stem cells. Nature 2001; 414: 92-97.

[15] Thompson JA, Itskovitz-Eldor J, Shapiro SS, et al. Embryonic stem cell lines derived from human blastocysts. Science 1998; 282: 1145-1147.

[16] Mitalipova M, Calhoun J, Shin S, et al. Human embryonic stem cell lines derived from discarded embryos. Stem Cells 2003; 21: 521-526

[17] Prusa AR, Morton E, Rosner M, et al. Oct-4-expressing cells in human amniotic fluid: a new source for stem cell research? Hum Reprod 2003; 18: 1489-1493.

[18] Milunsky A. Amniotic fluid cell culture. Genetic Disorder and the Fetus. Plenum Press, New York, 1979; pp. 75-84

[19] Hoehn H, Salk D. Morphological and biochemical heterogeneity of amniotic fluid cells in culture. Methods Cell Biol 1982; 26: 11-34.

[20] Gosden CM. Amniotic fluid cell types and cell culture. Br Med Bull 1983; 39: 348-354.

[21] Sakuragawa N, Ramasamy T, Mizuguchi M, et al. Expression of markers for both neuronal and glial cells in human amniotic epithelial cells. Neurosci Lett 1996; 209: 9-12.

[22] Bhattacharya B, Miura T, Brandenberger R, et al. Gene expression in human embryonic stem cell lines: unique molecular signature. Blood 2004; 103: 2956-2964

[23] Ohta Y, Tsutsumi K, Kikuchi K, et al. Two distinct human cervical epithelial cell lines established after transfection with human papillomavirus 16 DNA. J Cancer Res 1997; 88: 644-651.

[24] Kunimura C, Kikuchi K, Ahmed N, et al. Telomerase activity in a specific cell subset co-expressing integrin $\beta 1 /$ EGFR but not p75NGFR/bcl2/integrin $\beta 4$ in normal human epithelial cells. Oncogene 1998; 17: 187-197.
[25] Wei JP, Zhang TS, Kawa S, et al. Human amnion-isolated cells normalize blood glucose in streptozotocin-induced diabetic mice.Cell. Transplantation 2003; 12: 545-552.

[26] Hokari S, Miura K, Koyama I, et al. A restriction endonuclease assay for expression of human amylase isozymes. Clin Chim Acta 2002; 322: 113-116.

[27] Horii A, Emi M, Nishida T, et al. Primary structure of human pancreatic alpha-amylase gene: its comparison with human salivary alpha-amylase gene. Gene 1987; 60: 57-64.

[28] Hansis C, Grifo J, Krey LC, et al. Oct4 expression in inner cells mass and trophectoderm of human blastocysts. Mol Hum Reprod 2000; 6: 6999-1004.

[29] Simmonsson S, Gurdon J. DNA methylation is necessary for the epigenetic reprogramming of somatic nuclei. Nature CB 2004; 6 : 984-2004.

[30] Gurdon JB, Bryne JA, Simmonsson S. Nuclear reprogramming and stem cell creation. Proc Natl Acad Sci USA 2003; 100: 11819 11822

[31] Monk M, Holding C. Human embryonic genes re-expressed in cancer cells. Oncogene 2001; 56: 8085-8091.

[32] Chambers I, Smith A. Self-renewal of teratocarcinoma and embryonic stem cells. Oncogene 2004; 43: 7150-7160.

[33] Itoh H, Sagawa N, Hasegawa M, et al. Brain natriuretic peptide is present in human amniotic fluid and is secreted from amnion cells. J Clin Endocrinol Metab 1993; 76: 907-911.

[34] Kaviani A, Dario O F. The Amniotic Fluid as a Source of Cells for Fetal Tissue Engineering. J Pediatr Surg 2001; 36: 1662-1665.

[35] Wang DY, Hsueh YJ, Yang VC, et al. Propagation and phenotypic preservation of rabbit limbal epithelial cells on amniotic membrane. Invest Orhthalmol Vis Sci 2003; 44: 4698-4704.

[36] Yuge I, Takumi Y, Koyabu K, et al. Transplanted human amniotic epithelial cells express connexin 26 and $\mathrm{Na}-\mathrm{K}$-adenosine triphosphatase in the inner ear. Transplantation 2004; 77: 1452-1454.

[37] Yasumoto S, Kunimura C, Kikuchi K, et al. Telomerase activity in normal human epithelial cells. Oncogene 1996; 13: 433-439.

[38] Masutomi K, Yu EY, Khurts S, et al. Telomerase maintains telomere structure in normal human cells. Cell 2003; 114: 241-253.

[39] Kubota H, Reid LM. Clonogenic hepatoblasts,common precursors for hepatocytic and biliary lineages,are lacking classical major histocompatibility complex class I antigen. Proc Natl Acad Sci USA 2000; 97: 12132-12137.

[40] Lumelsky N, Blondel O, Laeng P, et al. Differentiation of embryonic stem cells to insulin-secreting structures similar to pancreatic islets. Science 2001; 292: 1389-1394.

[41] Tanaka S, Kunath T, Hadjantonakis AK, et al. Promotion of trophoblast stem cell proliferation by FGF4. Science 1998; 282: 2072 2075 . 